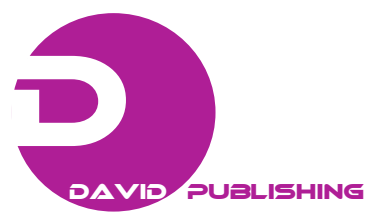

\title{
Seroprevalence Survey of Toxoplasma gondii in Swine in Northern Japan
}

\author{
Kanako Yamanouchi*, Kazuyuki Kida, Masahiro Kumagai, Jun-ichi Sasaki and Takashi Inaba \\ Department of Parasitology, Hirosaki University School of Health Sciences 66-1 Honcho, Hirosaki 36-8564, Aomori Japan \\ *Corresponding author's e-mail: tnappa@cc.hirosaki-u.ac.jp
}

\begin{abstract}
Infection rate of Toxoplasma gondii in humans is estimated to be at $10 \%-15 \%$ in Japan, and that in the world is at $33 \%$, respectively. Most of the infected adults are asymptomatic attributed to the immune system's contribution. However, women newly infected with Toxoplasma gondii during pregnancy can pass the organisms to the fetus that leads later to congenital toxoplasmosis, miscarriage or stillbirth. Number of congenital toxoplasmosis in Japan is estimated to be more than 130 cases per year. Encephalopathy due to toxoplasmosis occurs at $10 \%$ in the AIDS patients as a representative leading cause of death. General routes of transmission of Toxoplasma gondii to humans are: ingestion of oocysts shed from the final host feline, ingestion of cysts in the muscle of infected livestock, or vertical transmission to the fetus through placenta. Serological survey on toxoplasmosis was carried out to determine infection rate in pigs in Northern Japan, because pork is one of the most commonly consumed meats in Japan. Number of subjects provided for analyses was 481 sera collected from the slaughter house (meat-processing plant) in Hirosaki, Japan. Criteria applied for analyses were the latex agglutination and ELISA using the Toxoplasma-specific IgG antibodies. Positive sera in pigs were at 6\% (29/481) in prevalence. Further, epidemiological distribution pattern on Toxoplasma gondii was created by referring for breeding and feeding conditions in the pig farms. The results obtained indicate that Toxoplasma gondii is still circulating among pigs in Northern Japan. It is necessary to note the pregnant woman who does not have the toxoplasma antibody especially.
\end{abstract}

Key words: Toxoplasma gondii, seroprevalence, swine, ELISA, latex agglutination. 